\title{
Particle Size is a Primary Determinant for Sigmoidal Kinetics of Nanoparticle Formation: A "disproof" of the Finkey-Watzky (F-W) nanoparticle nucleation and growth mechanism.
}

\author{
James D. Martin
}

Department of Chemistry, North Carolina State University, Raleigh, NC 27695-8204

Supporting Information
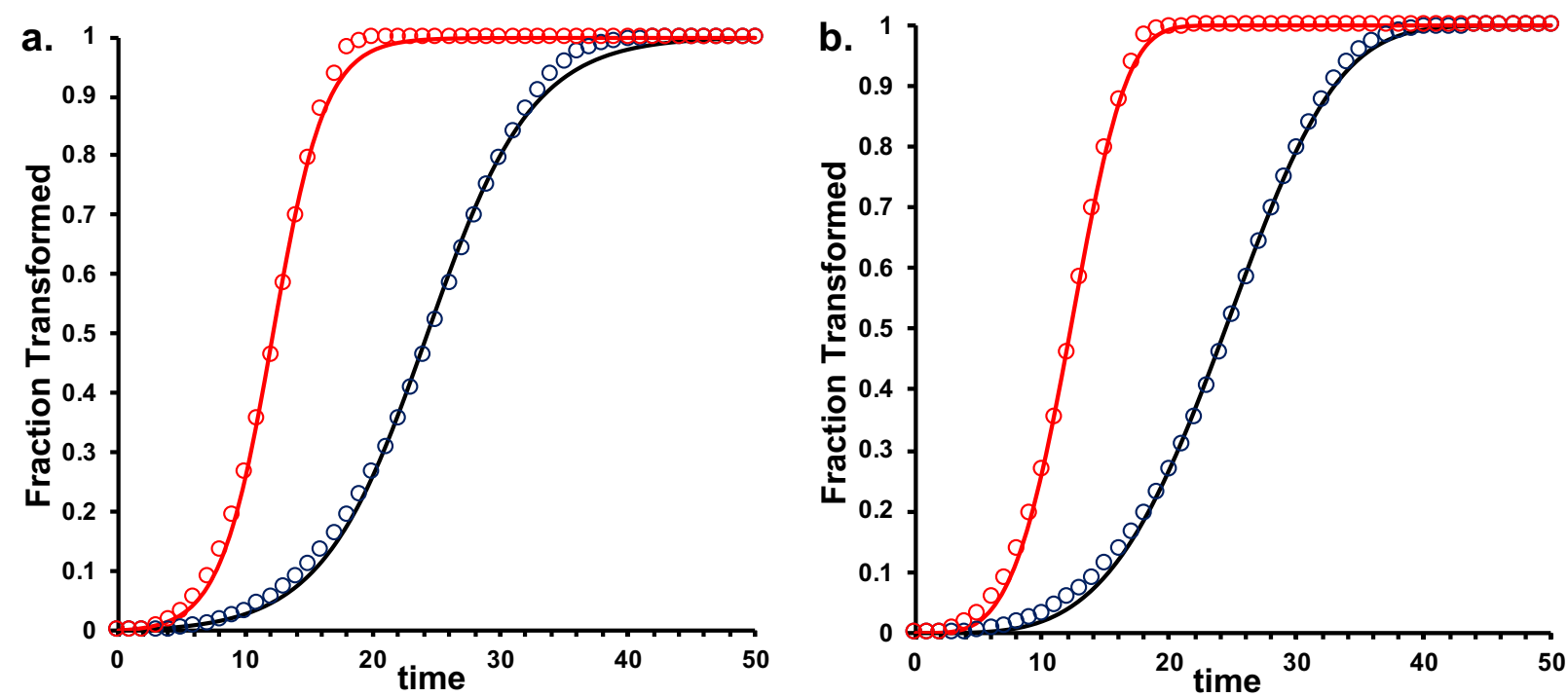

Figure S1. Normalized spherical particle growth as a function of time (Eq. $5, \mathrm{~kg}=0.02$ (blue) and 0.04 (red) distance/time) of a single spherical particle growing into a cubic volume. Solid lines represent fits to the entire transformation using a. the F-W and b. the M-KJMA models. 

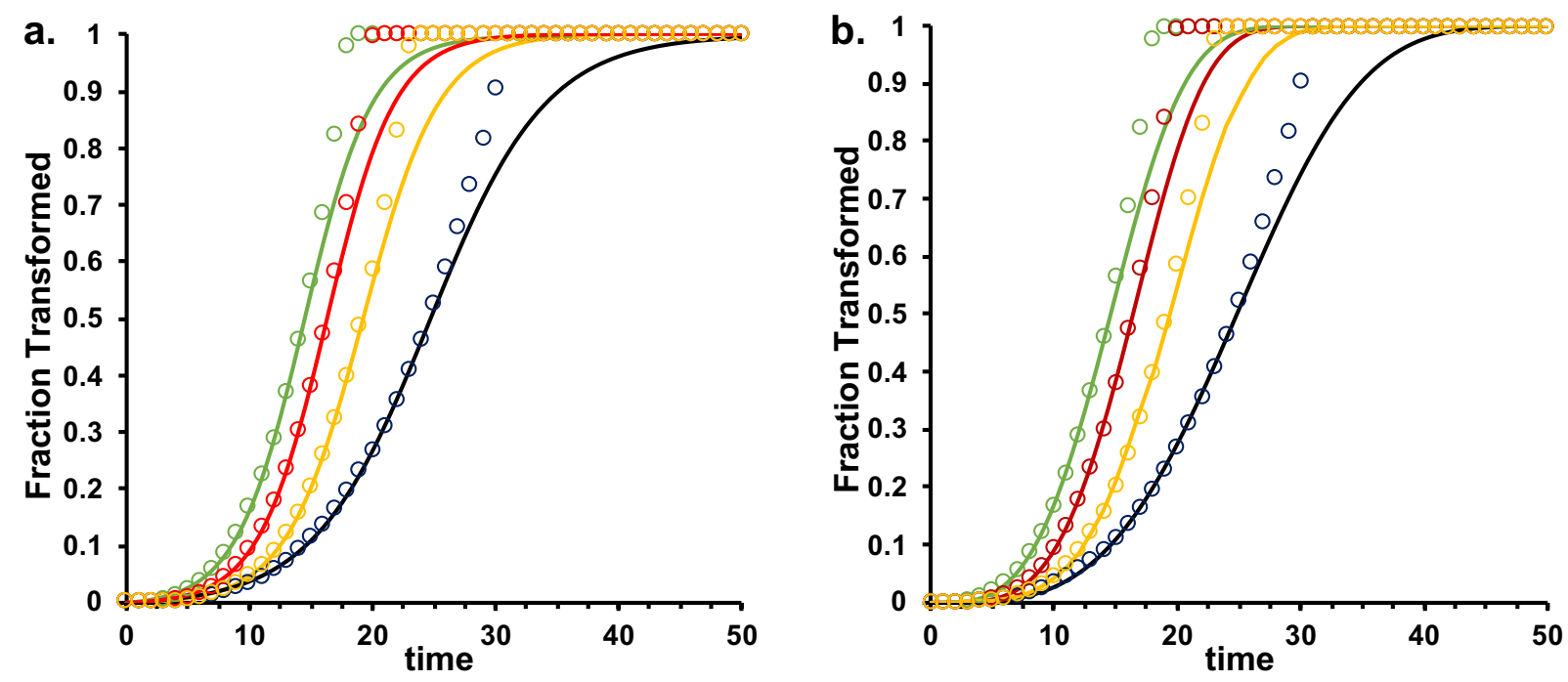

Figure S2. Spherical particle growth (Eq. 5, $\mathrm{kg}=0.02$ distance/time) of one (blue) or five (green) particles nucleated in a burst, or at continuous formation of five nuclei at rates of one nuclei/time step (red) and one nuclei/three time steps (yellow). Solid lines represent fits of the first $50 \%$ of growth using a. the F-W and $\mathbf{b}$. the M-KJMA models.
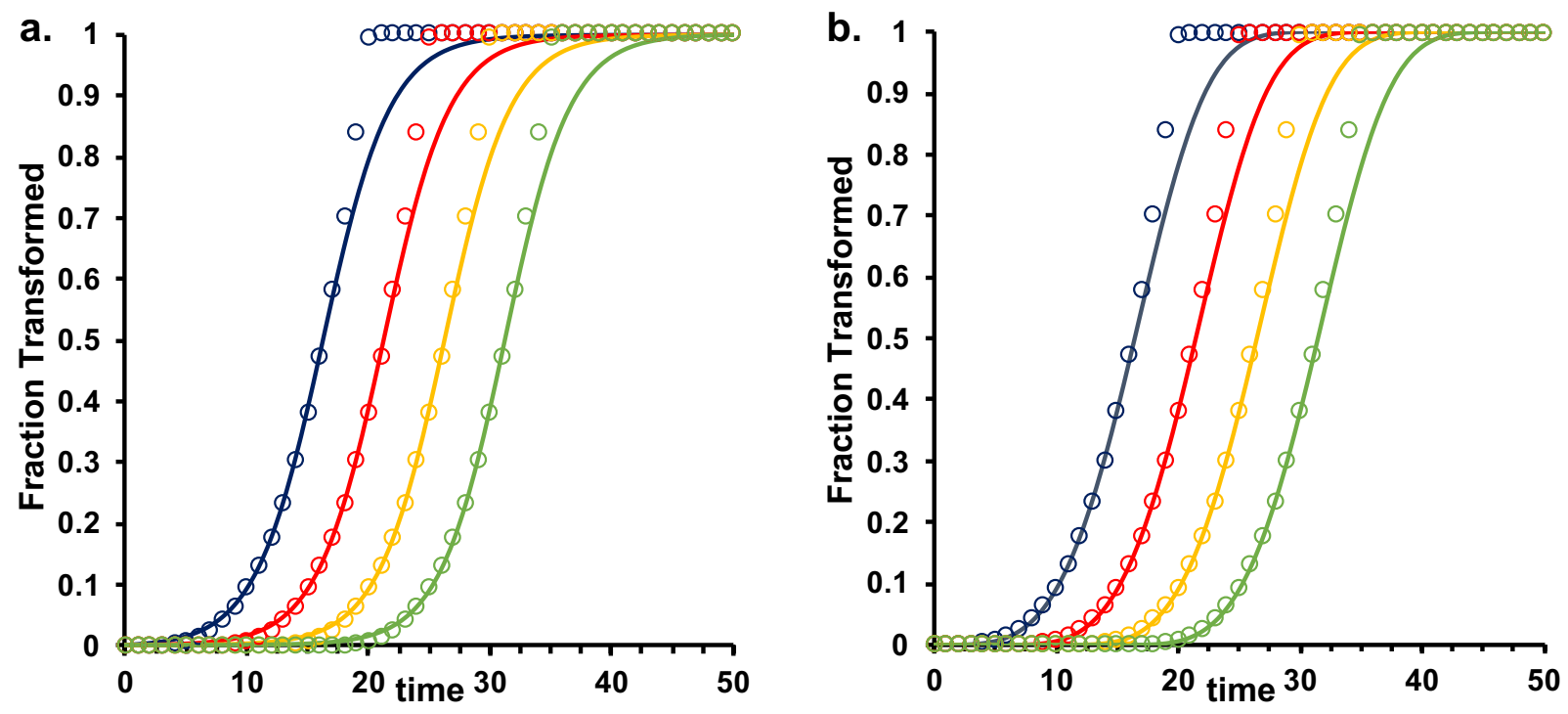

Figure S3. Spherical particle growth (Eq. 5, $\mathrm{kg}=0.02$ distance/time) with continuous formation of five nuclei at a rate of one nuclei/time step with an induction time of 0 (blue), 5 (red), 10 (yellow) and 15 (green) time steps. Solid lines represent fits of the first $50 \%$ of growth using a. the F-W and b. the M-KJMA models. 